\title{
On the Theory of Diffraction by a Composite Cylinder ${ }^{12}$
}

\author{
Ralph D. Kodis
}

\author{
(May 31, 1960)
}

\begin{abstract}
Formulas are developed for the diffracted field around a perfectly conducting cylinder with a dielectric sleeve of arbitrary thickness. These formulas represent the field due to a unit electric line source parallel to the cylinder (either inside or outside the dielectric sleeve) as a spectrum of radial eigenfunctions. It is shown that in each case the field in the region containing the source can be expressed as the sum of two terms, the first of which is a spectral representation of the field when the outer as well as the inner surface of the dielectric is a perfectly conducting boundary. The second term of the sum, which alone involves the properties of the dielectric, is an integral that converges rapidly at high frequencies. Using these general results, perturbation calculations are carried out for three limiting examples of plane wave scattering: (1) Thin dielectric; (2) low-refractive index; (3) very small surface curvature. In the latter approximation the correspondence with optical results is shown,
\end{abstract}

\section{Introduction}

The scattering of plane electromagnetic waves by cylindrical obstacles has received detailed theoretical treatment only in the two special cases for which the scalar wave function, $\Psi$, satisfies either the Dirichlet or the Neumann boundary condition at the cylindrical surface. In electromagnetic terms these problems correspond respectively to the scattering by a perfectly conducting cylinder of a wave whose electric or magnetic vector is parallel to the cylinder axis. For a cylindrical obstacle whose surface is not perfectly conducting a more complicated boundary condition of the type $\Psi+\alpha \partial_{n} \Psi=0$ is required (sometimes called an impedance boundary condition). In such cases the theory has not been so extensively elaborated. ${ }^{3}$

This paper is concerned with some of the theoretical features of the scattering problem associated with a cylindrical obstacle of this more general kind. In particular we shall examine the scattered wave produced by a dielectric cylinder of circular cross section having a perfectly conducting coaxial core. One aspect of this problem has been investigated experimentally by Tang $[1]^{4}$ for an incident wave with electric vector polarized parallel to the cylinder, and in the same paper the Fourier expansion of the wave function has been used for numerical calculations. It seems, however, that no general discussion of the characteristics of the wave solution has been given; it is this task which will now occupy our attention.

\section{Derivation of the Green's Function}

The cylinder which we shall consider (fig. 1) is composed of a perfectly conducting core of radius $a$ surrounded by a dielectric layer of inner radius $a$ and outer radius $b$. In the subsequent analysis the relative dielectric constant of the layer will be taken to be a real scalar greater than one, $\epsilon_{r}=\epsilon / \epsilon_{0}>1$. That is to say, the refractive index of the dielectric is a real number greater than that which characterizes the surrounding medium (air or vacuum). For a lossy or anisotropic dielectric the theory is unaffected except by the substitution throughout of a complex or tensor dielectric constant for $\epsilon$. Of course, the detailed calculation of field quantities will be much more complicated in such cases.

If the composite cylinder described above is excited by a harmonic line current parallel to its axis (electric line source, $\exp [-i \omega t])$, the task of finding the resultant electric field at

\footnotetext{
1 Contribution from the Division of Engineering, Brown University, Providence, R.I.

2 The research reported in this paper has been supported in part by the Office of Naval Research and the David W. Taylor Model Basin under contract Nonr 562(24) and in part by the Electronics Research Directorate of the Air Force Cambridge Research Center under contract A F $19-(604) 4561$.

${ }^{3}$ Certain aspects of such problems have been considered by J. R. Wait, Electromagnetic radiation from cylindrical structures, Chapter 16 (Pergamon Press, London, 1959).

${ }_{4}$ Figures in brackets indicate the literature references at the end of this paper.
} 


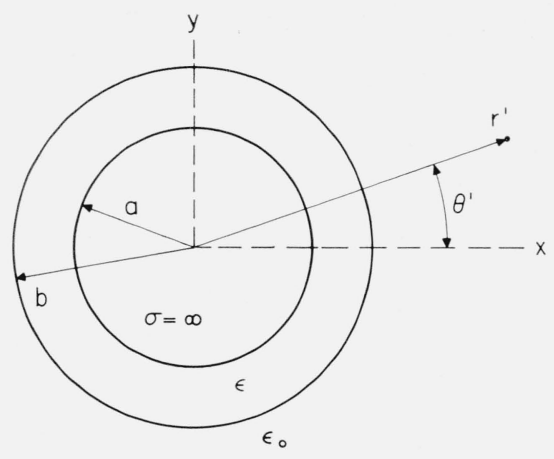

FiguRE 1. The composite cylinder and coordinate system.

every point outside the perfect conductor resolves itself into the determination of the Green's functions which satisfy appropriate partial differential equations and boundary conditions. For the moment we shall limit our considerations to the case in which the line source is outside the dielectric. Denoting coplanar position vectors relative to an origin on the cylinder axis by $\mathbf{r}^{\prime}$ and $\mathbf{r}$ for source and observation points, respectively, we require solutions in the $x y$-plane for the scalar wave equations

$$
\begin{aligned}
& \left(\nabla^{2}+k_{0}^{2}\right) G_{0}\left(\mathbf{r}, \mathbf{r}^{\prime}\right)=-\frac{1}{r} \delta\left(r-r^{\prime}\right) \delta\left(\theta-\theta^{\prime}\right) ; r, r^{\prime} \geq b \\
& \left(\nabla^{2}+k^{2}\right) G_{1}\left(\mathbf{r}, \mathbf{r}^{\prime}\right)=0 ; a \leq r \leq b, r^{\prime} \geq b
\end{aligned}
$$

subject to the following boundary conditions at $r=a$ and $r=b$ :

$$
\begin{aligned}
G_{1}\left(\mathbf{a}, \mathbf{r}^{\prime}\right) & =0 ; \\
G_{0}\left(\mathbf{b}, \mathbf{r}^{\prime}\right) & =G_{1}\left(\mathbf{b}, \mathbf{r}^{\prime}\right) ; \\
\partial_{r} G_{0}\left(\mathbf{b}, \mathbf{r}^{\prime}\right) & =\partial_{r} G_{1}\left(\mathbf{b}, \mathbf{r}^{\prime}\right) .
\end{aligned}
$$

Here the wave number, $k=\omega / v$, is related to the dielectric constant and the refractive index, $n$, of the dielectric according to

$$
k=k_{0} \sqrt{\epsilon_{r}}=n k_{0} .
$$

The mathematical problem defined by eqs (2.1) and (2.2) may be solved in a variety of ways. We shall employ a technique developed and discussed by Titchmarsh [2] and Marcuvitz [3] for separable equations. The procedure consists in constructing so-called characteristic functions which satisfy the related system of ordinary differential equations

$$
\begin{aligned}
{\left[\frac{d}{d r}\left(r \frac{d}{d r}\right)+r k_{0}^{2}-\frac{\lambda}{r}\right] f_{0}\left(r, r^{\prime}, \lambda\right) } & =-\delta\left(r-r^{\prime}\right), \\
{\left[\frac{d}{d r}\left(r \frac{d}{d r}\right)+r k^{2}-\frac{\lambda}{r}\right] f_{1}\left(r, r^{\prime}, \lambda\right) } & =0, \\
{\left[\frac{d^{2}}{d \theta^{2}}+\lambda\right] g\left(\theta, \theta^{\prime}, \lambda\right) } & =-\delta\left(\theta-\theta^{\prime}\right) .
\end{aligned}
$$

These equations are found from (2.1) by separating the polar variables $(r, \theta)$ with $\lambda$ taking the role of separation constant. In addition to (2.4) the functions $f_{0}$ and $f_{1}$ are required to satisfy boundary conditions corresponding to (2.2), while the angular function $g$ is made unique by certain convergence conditions to be specified. Once the characteristic functions have been explicitly determined from (2.4) and the boundary conditions, the Green's functions required by (2.1) are obtained by an integration in the complex $\lambda$-plane. For it can be verified that if 
$C$ is a circular contour of infinite radius in this plane, then

$$
\begin{aligned}
& \frac{1}{2 \pi i} \oint_{C} d \lambda f\left(r, r^{\prime}, \lambda\right)=r \delta\left(r-r^{\prime}\right), \\
& \frac{1}{2 \pi i} \oint_{C} d \lambda g\left(\theta, \theta^{\prime} \lambda\right)=\delta\left(\theta-\theta^{\prime}\right) .
\end{aligned}
$$

With the help of (2.4) and (2.5) direct substitution in (2.1) will show that the required Green's function is

$$
G\left(\mathbf{r}, \mathbf{r}^{\prime}\right)=\frac{1}{2 \pi i} \int_{\Gamma} d \lambda f\left(r, r^{\prime}, \lambda\right) g\left(\theta, \theta^{\prime}, \lambda\right)
$$

where $\Gamma$ is a contour which encloses the singularities of one of the functions $f$ or $g$, the sense of the contour being given either by (2.5a) or (2.5b) as the case may be.

In carrying out the procedure outlined above, we shall begin by considering the characteristic function which satisfies $(2.4 \mathrm{c})$. It has the form

$$
\left.g\left(\theta, \theta^{\prime}, \lambda\right)=A e^{i \nu(\theta}>^{-\theta}<\right),
$$

where $\nu^{2}=\lambda$ and $\theta>\theta<$ are respectively the larger and the smaller of the two variables $\theta$ and $\theta^{\prime}$. The positive exponential solution has been chosen to ensure the regularity of $g$ for all values of $\lambda$ on the principal sheet of the two-sheeted $\lambda$ surface. On this sheet $\operatorname{Im} \nu>0$, and since

$$
0<\arg \nu=\arg \sqrt{\lambda}<\pi
$$

the region of regularity corresponds to the upper half of the $\nu$-plane. The constant $A$ in (2.7) is determined by the fact that $d g / d \theta$ has unit discontinuity at $\theta=\theta^{\prime}$ for a delta function source. We thus find from $(2.4 \mathrm{c})$ that the characteristic function is

$$
\left.g\left(\theta, \theta^{\prime}, \lambda\right)=\frac{i}{2 \nu} e^{i \nu(\theta}>^{-\theta}<\right)=\frac{i}{2 \nu} e^{i \nu\left|\theta-\theta^{\prime}\right|}, \quad 0<\arg \nu<\pi .
$$

It is easy to verify that (2.9) satisfies (2.5b). Since the only singularity of $g\left(\theta, \theta^{\prime}, \lambda\right)$ is a branch point at $\lambda=0$, the clockwise contour $C$ may be deformed to an integral around the branch cut which is conveniently taken along the positive real axis of the $\lambda$-plane. If the deformed contour is now mapped onto the real axis of the $\nu$-plane by the transformation $\lambda=\nu^{2}$, the resulting integral is seen to be a representation of the $\delta$-function. Thus

$$
\frac{1}{2 \pi i} \oint d \lambda g\left(\theta, \theta^{\prime}, \lambda\right)=\frac{1}{2 \pi} \int_{-\infty}^{\infty} d \nu e^{i \nu\left|\theta-\theta^{\prime}\right|}=\delta\left(\theta-\theta^{\prime}\right) \text {. }
$$

The characteristic Green's functions which satisfy (2.4a) and (2.4b) are constructed in a similar fashion but from linear combinations of Bessel functions of order $\nu=\sqrt{\lambda}$. At large distances from the source, $r \gg r^{\prime}$, they must represent outgoing waves. Furthermore, the interior function must vanish at the conducting surface $r=a$, and the exterior function must behave like a "standing wave" in the region $r^{\prime}>r \geq b$. In terms of Hankel functions of the first and second kinds the most general expressions which exhibit these characteristics are

$$
\begin{aligned}
& f_{0}\left(r, r^{\prime}, \lambda\right)=B_{0}\left[H_{\nu}^{(1)}\left(k_{0} b\right) H_{\nu}^{(2)}\left(k_{0} r_{<}\right)-D H_{\nu}^{(2)}\left(k_{0} b\right) H_{\nu}^{(1)}\left(k_{0} r_{<}\right)\right] H_{\nu}^{(1)}\left(k_{0} r_{>}\right) ; r, r^{\prime} \geq b, \\
& f_{1}\left(r, r^{\prime}, \lambda\right)=B_{1}\left[H_{\nu}^{(1)}(k a) H_{\nu}^{(2)}\left(k r_{<}\right)-H_{\nu}^{(2)}(k a) H_{\nu}^{(1)}\left(k r_{<}\right)\right] H_{\nu}^{(1)}\left(k_{0} r_{>}\right) ; a \leq r \leq b, r^{\prime} \geq b .
\end{aligned}
$$

Two of the three integration constants appearing in (2.10) and (2.11) are determined by the pair of linear algebraic equations which express the continuity of the radial function and its first derivative at $r=b$. The remaining constant is fixed by the jump in $r \frac{d}{d r} f_{0}\left(r, r^{\prime}, \lambda\right)$ at $r=r^{\prime}$. 
Making use of the Wronskian relation

$$
H_{\nu}^{(1)}\left(\beta_{c}\right) H_{\nu}^{(2) '}\left(\beta_{0}\right)-H_{\nu}^{(1) \prime}\left(\beta_{0}\right) H_{\nu}^{(2)}\left(\beta_{0}\right)=-\frac{4 i}{\pi \beta_{0}},
$$

and introducing the notation

$$
\begin{gathered}
\alpha=k a, \quad \beta=k b, \quad \beta_{0}=k_{0} b ; \\
{ }^{5} C_{\nu}(k r)=H_{\nu}^{(1)}(\alpha) H_{\nu}^{(2)}(k r)-H_{\nu}^{(2)}(\alpha) H_{\nu}^{(1)}(k r), \\
C_{\nu}^{\prime}(k r)=H_{\nu}^{(1)}(\alpha) H_{\nu}^{(2) '}(k r)-H_{\nu}^{(2)}(\alpha) H_{\nu}^{(1)^{\prime}}(k r) ;
\end{gathered}
$$

and

$$
X_{\nu}^{(1,2)}=\beta \frac{C_{\nu}^{\prime}(\beta)}{C_{\nu}(\beta)}-\beta_{0} \frac{H_{\nu}^{(1,2) \prime}\left(\beta_{0}\right)}{H_{\nu}^{(1,2)}\left(\beta_{0}\right)}
$$

we find that the characteristic function for an exterior source at $r^{\prime}$ is

$$
\begin{aligned}
& f_{0}\left(r, r^{\prime}, \lambda\right)=\frac{\pi i}{4}\left[H_{\nu}^{(2)}\left(k_{0} r_{<}\right)-\frac{H_{\nu}^{(2)}\left(\beta_{0}\right)}{H_{\nu}^{(1)}\left(\beta_{0}\right)} \frac{X_{\nu}^{(2)}}{X_{\nu}^{(1)}} H_{\nu}^{(1)}\left(k_{0} r_{<}\right)\right] H_{\nu}^{(1)}\left(k_{0} r_{>}\right), \quad r \geq b ; \\
& f_{1}\left(r, r^{\prime}, \lambda\right)=\frac{C_{\nu}(k r) H_{\nu}^{(1)}\left(k_{0} r^{\prime}\right)}{H_{\nu}^{(1)}\left(\beta_{0}\right) C_{\nu}(\beta) X_{\nu}^{(1)}}, \quad r \leq b .
\end{aligned}
$$

A direct calculation shows that these functions satisfy (2.5a).

It remains to determine $G\left(\mathbf{r}, \mathbf{r}^{\prime}\right)$ from (2.6). First we note that the only singularities of $f_{0}$ and $f_{1}$ in the $\lambda$-plane are simple poles at the set of complex values, $\lambda_{j}$, for which

$$
X_{v_{j}}^{(1)}=0 .
$$

That these functions have no branch point is easy to verify. With the relations,

$$
H_{-\nu}^{(1)}=e^{i \nu \pi} H_{\nu}^{(1)} \text { and } H_{-\nu}^{(2)}=e^{-i \nu \pi} H_{\nu}^{(2)},
$$

we learn that

$$
C_{-\nu}=C_{\nu} \quad \text { and } \quad X_{-\nu}=X_{\nu}
$$

It follows that $f_{0}$ and $f_{1}$ are both single-valued over the entire $\lambda$-plane. In accordance with (2.6), we now form the integral

$$
G\left(\mathbf{r}, \mathbf{r}^{\prime}\right)=\frac{1}{2 \pi i} \int_{\Gamma} \frac{i d \lambda}{2 \nu} f\left(r, r^{\prime}, \lambda\right) e^{i \nu\left|\theta-\theta^{\prime}\right|}
$$

where the contour $\Gamma$ is chosen to enclose the singularities of $f$ in a positive sense. Since the product function has a branch point at $\lambda=0, \Gamma$ must be indented around the branch cut, which we shall take along the real axis as shown in figure 2. In the $\nu$-plane the resulting contour maps to $\gamma$. By virtue of (2.8) and the asymptotic behavior of the Hankel functions, the contribution to (2.19) from the semicircular segment of $\gamma$ may be made arbitrarily small if the radius of the arc is made large enough. In this way we see that both a discrete and a continuous representation of $G$ are possible,

$$
G\left(\mathbf{r}, \mathbf{r}^{\prime}\right)=\sum \operatorname{Res}\left(\nu=\nu_{\jmath}\right)=\frac{1}{2 \pi} \int_{-\infty}^{\infty} d \nu f\left(r, r^{\prime}, \lambda\right) e^{i \nu\left|\theta-\theta^{\prime}\right|} .
$$

\footnotetext{
${ }^{5}$ It would be more explicit to write $C_{\nu}(\alpha, k r)$, but since subsequent operations on $C_{\nu}$ affect it only as a function of $k r$, it is perhaps clearer to use the abbreviated notation.
} 

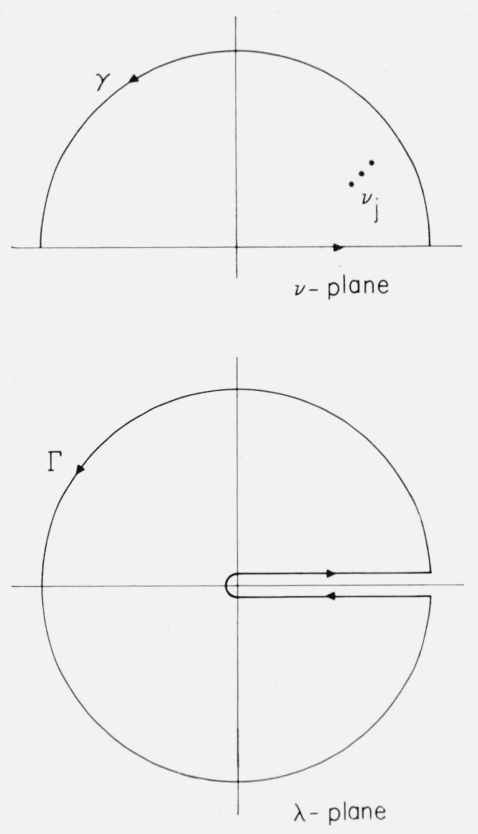

FIgure 2. The complex $\nu$ and $\lambda$ planes.

\section{The Discrete Spectrum}

The discrete representation of the Green's function is obtained by evaluating the residues of $f\left(r, r^{\prime}, \lambda\right)$ from (2.16) and (2.17). A more symmetrical form results if one makes use of the relation

$$
X_{\nu}^{(2)}=X_{\nu}^{(1)}+\frac{4 i}{\pi} \frac{1}{H_{\nu}^{(1)}\left(\beta_{0}\right) H_{\nu}^{(2)}\left(\beta_{0}\right)}
$$

which, at $\nu=\nu_{j}$, becomes simply

$$
X_{j}^{(2)}=\frac{4 i}{\pi} \frac{1}{H_{i}^{(1)}\left(\beta_{0}\right) H_{i}^{(2)}\left(\beta_{0}\right)},
$$

where the subscript indicates the $j^{\text {th }}$ root of (2.18). With this change the residue sums corresponding to (2.19) form discrete spectral representations of the total field due to an exterior line source at $r^{\prime} \geq b$. They are

$$
\begin{aligned}
& G_{0}\left(\mathbf{r}, \mathbf{r}^{\prime}\right)=i \sum_{j} \frac{1}{\partial_{v} X_{j}^{(1)}} \frac{H_{i}^{(1)}\left(k_{0} r\right) H_{j}^{(1)}\left(k_{0} r^{\prime}\right)}{H_{j}^{(1)}\left(\beta_{0}\right) H_{j}^{(1)}\left(\beta_{0}\right)} e^{i \nu_{j}\left|\theta-\theta^{\prime}\right|} ; \quad r \geq b, \\
& G_{1}\left(\mathbf{r}, \mathbf{r}^{\prime}\right)=i \sum_{j} \frac{1}{\partial_{v} X_{j}^{(1)}} \frac{C_{j}(k r) H_{i}^{(1)}\left(k_{0} r^{\prime}\right)}{C_{j}(\beta) H_{j}^{(1)}\left(\beta_{0}\right)} e^{i v_{j}\left|\theta-\theta^{\prime}\right|} ; \quad a \leq r \leq b .
\end{aligned}
$$

It is now clear from considerations of symmetry that if the line source is inside the dielectric, $a \leq r^{\prime} \leq b$, than

$$
\begin{aligned}
G_{0}\left(\mathbf{r}, \mathbf{r}^{\prime}\right) & =i \sum_{j} \frac{1}{\partial_{\nu} X_{j}^{(1)}} \frac{H_{j}^{(1)}\left(k_{0} r\right) C_{j}\left(k r^{\prime}\right)}{H_{j}^{(1)}\left(\beta_{0}\right) C_{j}(\beta)} e^{i \nu_{j}\left|\theta-\theta^{\prime}\right|} ; \quad r \geq b, \\
G_{1}\left(\mathbf{r}, \mathbf{r}^{\prime}\right) & =i \sum \frac{1}{\partial_{\nu} X_{i}^{(1)}} \frac{C_{j}(k r) C_{j}\left(k r^{\prime}\right)}{C_{j}(\beta) C_{j}(\beta)} e^{i \nu_{j}\left|\theta-\theta^{\prime}\right|} ; \quad a \leq r \leq b .
\end{aligned}
$$

It is noteworthy that the representations (3.3a) and (3.4b), with source and observation points 
in the same region, are unaffected by an interchange of the variables $r$ and $r^{\prime}$. If $r$ and $r^{\prime}$ are in different regions, however, their interchange turns (3.3b) into (3.4a) and vice versa.

The radial eigenfunction expansions derived above have certain characteristic features which should be mentioned. For fixed $r$ and $r^{\prime}$ each term of the series represents (in the terminology of Franz) a "creeping wave" propagating in the $\theta$-direction with exponentially damped amplitude. Since $\theta$ covers the interval $(-\infty, \infty)$, the total field at any point is the sum of the contributions at $\theta \pm 2 m \pi$ for all integral values of $m$. At high frequencies, however, the imaginary part of $\nu$ is a large positive number so that all terms other than the one for which $m=0$ are exponentially small. Asymptotically, (3.3) and (3.4) represent the total field in the range $0<\left|\theta-\theta^{\prime}\right|<2 \pi$. It is to be expected, moreover, that the set of $\nu_{j}$ are ordered with increasingly large imaginary parts so that if $\left|\theta-\theta^{\prime}\right| \neq 0$, only the first term of each residue expansion will contribute significantly to the field at high enough frequencies. However, the problem of finding even the first root of (2.18) would seem to be a formidable one.

\section{The Continuous Spectrum}

An alternative representation of the Green's function as an integral over a continuous spectrum is obtained from (2.19), using (2.16) and (2.17). Since $g\left(\theta, \theta^{\prime}, \lambda\right)$ has a branch point singularity at $\lambda=0$, the contour integral becomes

$$
G\left(\mathbf{r}, \mathbf{r}^{\prime}\right)=\frac{1}{4 \pi} \bigodot_{\leftarrow}^{\vec{\leftarrow}} \frac{d \lambda}{\nu} e^{i \nu\left|\theta-\theta^{\prime}\right|} f\left(r, r^{\prime}, \lambda\right),
$$

where the indicated path is around a branch cut along the real axis of the $\lambda$-plane. With the help of (3.1) and the change of variable, $\lambda=\nu^{2}$, we find from (4.1) that

$$
\begin{gathered}
G_{0}\left(\mathbf{r}, \mathbf{r}^{\prime}\right)=\frac{i}{8} \int_{-\infty}^{\infty} d \nu\left[H_{\nu}^{(1)}\left(\beta_{0}\right) H_{\nu}^{(2)}\left(k_{0} r_{<}\right)-H_{\nu}^{(2)}\left(\beta_{0}\right) H_{\nu}^{(1)}\left(k_{0} r_{<}\right)\right] \frac{H_{\nu}^{(1)}\left(k_{0} r_{>}\right)}{H_{\nu}^{(1)}\left(\beta_{0}\right)} e^{i \nu \mid \theta-\theta^{\prime}} \\
\quad+\frac{1}{2 \pi} \int_{-\infty}^{\infty} \frac{d \nu}{X_{\nu}^{(1)}} \frac{H_{\nu}^{(1)}\left(k_{0} r_{<}\right) H_{\nu}^{(1)}\left(k_{0} r_{>}\right)}{H_{\nu}^{(1)}\left(\beta_{0}\right) H_{\nu}^{(1)}\left(\beta_{0}\right)} e^{i \nu\left|\theta-\theta^{\prime}\right|} ; \quad r, r^{\prime} \geq b
\end{gathered}
$$

Similarly the field inside the dielectric is

$$
G_{1}\left(\mathbf{r}, \mathbf{r}^{\prime}\right)=\frac{1}{2 \pi} \int_{-\infty}^{\infty} \frac{d \nu}{X_{\nu}^{(1)}} \frac{C_{\nu}(k r) H_{\nu}^{(1)}\left(k_{0} r^{\prime}\right)}{C_{\nu}(\beta) H_{\nu}^{(1)}\left(\beta_{0}\right)} e^{i \nu\left|\theta-\theta^{\prime}\right|} ; \quad r^{\prime} \geq b, a \leq r \leq b .
$$

In this form the Green's function of (4.2) has some interesting features. The first term satisfies all the conditions associated with the field of an electric line source in the vicinity of a perfectly conducting cylinder of radius $b$ when the medium is characterized by the propagation number $k_{0}$. One way to see this is to close the integration contour in the upper half of the $\nu$-plane. The residue sum that results is

$$
G_{c}\left(\mathbf{r}, \mathbf{r}^{\prime}\right)=\frac{\pi}{4} \sum_{p} \frac{H_{p}^{(2)}\left(\beta_{0}\right)}{\partial_{\nu} H_{p}^{(1)}\left(\beta_{0}\right)} H_{p}^{(1)}\left(k_{0} r_{<}\right) H_{p}^{(1)}\left(k_{0} r_{>}\right) e^{i \nu_{p}\left|\theta-\theta^{\prime}\right|}
$$

where

$$
H_{p}^{(1)}\left(\beta_{0}\right)=H_{\nu_{p}}^{(1) \prime}\left(\beta_{0}\right)=0
$$

From the Wronskian, (2.12), one finds that

$$
H_{p}^{(2)}\left(\beta_{0}\right)=\frac{4 i}{\pi \beta_{0} H_{p}^{(1) \prime}\left(\beta_{0}\right)},
$$


so that (4.4) can be rearranged in the form

$$
\begin{aligned}
G_{c}\left(\mathbf{r}, \mathbf{r}^{\prime}\right) & =\frac{i}{\beta_{0}} \sum_{p} \frac{H_{p}^{(1)}\left(k_{0} r\right) H_{p}^{(1)}\left(k_{0} r^{\prime}\right)}{\partial_{\nu} H_{p}^{(1)}\left(\beta_{0}\right) H_{p}^{(1) \prime}\left(\beta_{0}\right)} e^{i \nu p\left|\theta-\theta^{\prime}\right|} \\
= & \frac{1}{2 \pi \beta_{0}} \int_{-\infty}^{\infty} d \nu \frac{H_{\nu}^{(1)}\left(k_{0} r\right) H_{\nu}^{(1)}\left(k_{0} r^{\prime}\right)}{H_{\nu}^{(1)}\left(\beta_{0}\right) H_{\nu}^{(1)}\left(\beta_{0}\right)} e^{i \nu\left|\theta-\theta^{\prime}\right|} .
\end{aligned}
$$

These are the results for a conducting cylinder which have been derived in other ways by $\mathrm{T}$. $\mathrm{Wu}[4]$ and by the author [5].

We see then that the field of a composite cylinder has as one part the creeping waves of a conducting cylinder. Only the second integral of (4.2) depends upon the parameters of the dielectric (thickness and refraction index) and this dependence resides in the factor $X_{\nu}^{(1)}$. Indeed as $a \rightarrow b, X_{\nu}^{(1)}$ grows without limit and the integral vanishes, leaving the Green's function for a conducting cylinder of radius $b$. On the other hand, as $k \rightarrow k_{0}$ the limiting value of the second integral of $(4.2)$ is

$$
-\frac{i}{8} \int_{-\infty}^{\infty} d \nu\left[\frac{H_{\nu}^{(2)}\left(\alpha_{0}\right)}{H_{\nu}^{(1)}\left(\alpha_{0}\right)}-\frac{H_{v}^{(2)}\left(\beta_{0}\right)}{H_{\nu}^{(1)}\left(\beta_{0}\right)}\right] H_{\nu}^{(1)}\left(k_{0} r_{<}\right) H_{\nu}^{(1)}\left(k_{0} r_{>}\right) e^{i \nu\left|\theta-\theta^{\prime}\right|}
$$

and on substituting we find that the Green's function reduces to that for a conducting cylinder of radius $a$. These limiting forms will be used later for perturbation calculations.

When the line source is inside the dielectric sleeve, the derivation of the Green's function proceeds in a similar way. We begin with characteristic functions for the radial equations having the form

$$
\begin{aligned}
& h_{0}\left(r, r^{\prime}, \lambda\right)=A_{0} C_{\nu}\left(k r_{<}\right) H_{\nu}^{(1)}\left(k_{0} r_{>}\right) ; \quad a \leq r^{\prime} \leq b, \quad r \geq b, \\
& h_{1}\left(r, r^{\prime}, \lambda\right)=A_{1} C_{\nu}\left(k r_{<}\right)\left[H_{\nu}^{(2)}\left(k r_{>}\right)+B H_{\nu}^{(1)}\left(k r_{>}\right)\right] ; \quad a \leq r, r^{\prime} \leq b .
\end{aligned}
$$

Both functions satisfy the boundary condition at $r_{<}=a$, and $h_{0}$ represents an outgoing cylindrical wave as $r>\rightarrow \infty$. The constants can be evaluated as before, and after a certain amount of manipulation, we find that

$$
\begin{aligned}
& h_{0}\left(r, r^{\prime}, \lambda\right)=\frac{1}{X_{\nu}^{(1)}} \frac{C_{\nu}\left(k r^{\prime}\right) H_{\nu}^{(1)}\left(k_{0} r\right)}{C_{\nu}(\beta) H_{\nu}^{(1)}\left(\beta_{0}\right)} \\
& h_{1}\left(r, r^{\prime}, \lambda\right)=-\frac{\pi i}{4} \frac{C_{\nu}\left(k r_{<}\right)}{C_{\nu}(\beta)}\left[H_{\nu}^{(1)}(\beta) H_{\nu}^{(2)}\left(k r_{>}\right)-H_{\nu}^{(2)}(\beta) H_{\nu}^{(1)}\left(k r_{>}\right)\right]+\frac{1}{X_{\nu}^{(1)}} \frac{C_{\nu}\left(k r_{<}\right) C_{\nu}\left(k r_{>}\right)}{C_{\nu}(\beta) C_{\nu}(\beta)} .
\end{aligned}
$$

The exterior function $h_{0}\left(r, r^{\prime}, \lambda\right)$ of (4.11) corresponds exactly to $f_{1}$ of (2.17) after an interchange of the variables $r$ and $r^{\prime}$, so the related Green's function corresponds to (4.3). When both source and observation points are inside the dielectric, the convolution of $h_{1}\left(r, r^{\prime}, \lambda\right)$ with $(2.9)$ around the branch cut of $g\left(\theta, \theta^{\prime}, \lambda\right)$ leads to the following integral representation of the Green's function:

$$
\begin{aligned}
G_{1}\left(\mathbf{r}, \mathbf{r}^{\prime}\right)=-\frac{i}{8} \int_{-\infty}^{\infty} d \nu \frac{C_{\nu}\left(k r_{<}\right)}{C_{\nu}(\beta)}\left[H_{\nu}^{(1)}(\beta) H_{\nu}^{(2)}\left(k r_{>}\right)-H_{\nu}^{(2)}(\beta) H_{\nu}^{(1)}\left(k r_{>}\right)\right] e^{i \nu\left|\theta-\theta^{\prime}\right|} & \\
& +\frac{1}{2 \pi} \int_{-\infty}^{\infty} \frac{d \nu}{X_{\nu}^{(1)}} \frac{C_{\nu}\left(k r_{<}\right) C_{\nu}\left(k r_{>}\right)}{C_{\nu}(\beta) C_{\nu}(\beta)} e^{i \nu\left|\theta-\theta^{\prime}\right| .}
\end{aligned}
$$

The first term of this Green's function vanishes at $r<=a$ and $r=b$, and one can easily verify that it corresponds exactly to the field of an electric line source inside a dielectric filled coaxial transmission line with perfectly conducting walls. The second term represents the modification of that field due to radiation into the exterior medium. 
The residue sum associated with (4.11) has already been given in (3.4). To complete the discussion we shall write down the symmetrical representations for a coaxial line, analogous to (4.7) and (4.8). They follow from the convolution of the first term of (4.12) with $g\left(\theta, \theta^{\prime}, \lambda\right)$ around the simple poles located at the points of the $\lambda$-plane for which

$$
C_{\nu_{s}}(\beta)=C_{s}(\beta)=0 \text {. }
$$

It is found that

$$
\begin{aligned}
G_{c o}\left(\mathbf{r}, \mathbf{r}^{\prime}\right) & =-\frac{i}{\beta} \sum_{s} \frac{C_{s}(k r) C_{s}\left(k r^{\prime}\right)}{\partial_{\nu} C_{s}(\beta) C_{s}^{\prime}(\beta)} e^{i \nu_{s}\left|\theta-\theta^{\prime}\right|} \\
& =-\frac{1}{2 \pi \beta} \int_{-\infty}^{\infty} d \nu \frac{C_{\nu}(k r) C_{\nu}\left(k r^{\prime}\right)}{C_{\nu}(\beta) C_{\nu}^{\prime}(\beta)} e^{i \nu\left|\theta-\theta^{\prime}\right|}
\end{aligned}
$$

where we have made use of the Wronskian relations,

$$
H_{\nu}^{(1,2)}(k r) C_{\nu}^{\prime}(k r)-H_{\nu}^{(1,2)^{\prime}}(k r) C_{\nu}(k r)=-\frac{4 i}{\pi k r} H_{\nu}^{(1,2)}(\alpha) .
$$

\section{Plane-Wave Excitation}

In order to render more manageable the integrals appearing in (4.2) it is convenient at this stage to turn one's attention to special cases. Since the general class of problems associated with the scattering of a plane wave is of considerable interest, we shall first consider the question of reducing the field to that of an incident plane wave of unit amplitude. This may be done by considering the first term of (4.2), which we shall call $G_{b}$,

$$
G_{b}\left(\mathbf{r}, \mathbf{r}^{\prime}\right)=\frac{i}{8} \int_{-\infty}^{\infty} d \nu\left[H_{\nu}^{(2)}\left(k_{0} r_{<}\right)-\frac{H_{\nu}^{(2)}\left(\beta_{0}\right)}{H_{\nu}^{(1)}\left(\beta_{0}\right)} H_{\nu}^{(1)}\left(k_{0} r_{<}\right)\right] H_{\nu}^{(1)}\left(k_{0} r_{>}\right) e^{i \nu\left|\theta-\theta^{\prime}\right|}
$$

The Hankel functions, $H_{\nu}(\rho)$, grow as $\exp \left(\nu \ln \frac{2 \nu}{\rho}\right)$ when $\nu \rightarrow+\infty$, so the individual terms of this integral diverge and cannot be treated separately. However the divergences may be removed by first putting (5.1) in the form,

$$
G_{b}\left(\mathbf{r}, \mathbf{r}^{\prime}\right)=\frac{i}{4} \int_{0}^{\infty} d \nu\left[H_{\nu}^{(2)}\left(k_{0} r_{<}\right)-\frac{H_{\nu}^{(2)}\left(\beta_{0}\right)}{H_{\nu}^{(1)}\left(\beta_{0}\right)} H_{\nu}^{(1)}\left(k_{0} r_{<}\right)\right] H_{\nu}^{(1)}\left(k_{0} r_{>}\right) \cos \nu\left(\theta-\theta^{\prime}\right),
$$

and then making the substitution,

$$
H_{\nu}^{(2)}(\rho)=2 J_{\nu}(\rho)-H_{\nu}^{(1)}(\rho) .
$$

With these changes it is found that

$G_{b}\left(\mathbf{r}, \mathbf{r}^{\prime}\right)=\frac{i}{2} \int_{0}^{\infty} d \nu J_{\nu}\left(k_{0} r_{<}\right) H_{\nu}^{(1)}\left(k_{0} r_{>}\right) \cos \nu\left(\theta-\theta^{\prime}\right)-\frac{i}{2} \int_{0}^{\infty} d \nu \frac{J_{\nu}\left(\beta_{0}\right)}{H_{\nu}^{(1)}\left(\beta_{0}\right)} H_{\nu}^{(1)}\left(k_{0} r_{<}\right) H_{\nu}^{(1)}\left(k_{0} r_{>}\right) \cos \nu\left(\theta-\theta^{\prime}\right)$.

Each of these integrals is absolutely convergent.

To establish a correspondence between (5.3) and the required plane wave solution, we follow Wu's procedure and sum the creeping waves for the first term. Then by interchanging the order of summation and integration (or by invoking the Poisson summation formula) it can be shown that

$$
\frac{i}{2} \sum_{m=-\infty}^{\infty} \int_{0}^{\infty} d \nu J_{\nu}\left(k_{0} r_{<}\right) H_{\nu}^{(1)}\left(k_{0} r_{>}\right) \cos \nu\left(\theta-\theta^{\prime}+2 m \pi\right)=\frac{i}{4} H_{0}^{(1)}\left(k_{0}\left|\mathbf{r}_{>}-\mathbf{r}_{<}\right|\right),
$$


where the function on the right is just the free space Green's function representing a unit line source. If we now let $r>\rightarrow \infty$ and make use of the asymptotic form,

$$
H_{\nu}^{(1)}(\rho) \sim \sqrt{\frac{2}{\pi \rho}} e^{i\left(\rho-\frac{\pi}{4}\right)} e^{-i v \frac{\pi}{2}}
$$

both sides of (5.4) can be normalized to an incident plane wave of unit amplitude by multiplying with the factor $-4 i(2)^{-1 / 2}\left(k_{0} r_{>}\right) \exp -i\left(k_{0} r_{>}-\pi / 4\right)$. Making these changes in (5.3) and setting $\theta^{\prime}=0$ gives the total field, $\psi_{b}(\mathbf{r})$, when a plane wave from the right is scattered by a conducting cylinder of radius $b$. This field is

$$
\psi_{b}(\mathbf{r})=2 \int_{0}^{\infty} d \nu J_{\nu}\left(k_{0} r\right) e^{-i \nu \frac{\pi}{2}} \cos \nu \theta-2 \int_{0}^{\infty} d \nu \frac{J_{\nu}\left(\beta_{0}\right)}{H_{\nu}^{(1)}\left(\beta_{0}\right)} H_{\nu}^{(1)}\left(k_{0} r\right) e^{-i \nu \frac{\pi}{2}} \cos \nu \theta .
$$

The procedure that led to (5.4) will also show that

$$
2 \sum_{m=-\infty}^{\infty} \int_{0}^{\infty} d \nu J_{\nu}\left(k_{0} r\right) e^{-i \nu \frac{\pi}{2}} \cos \nu(\theta+2 m \pi)=e^{-i k_{0} r \cos \theta} .
$$

When (4.2) has been normalized in accordance with (5.5), the total field outside a dielectric clad cylinder illuminated by a plane wave from the right becomes

$$
\psi(\mathbf{r})=\psi_{b}(\mathbf{r})+\frac{2}{\pi i} \int_{-\infty}^{\infty} \frac{d \nu}{X_{\nu}^{(1)}} \frac{H_{\nu}^{(1)}\left(k_{0} r\right)}{\left[H_{\nu}^{(1)}\left(\beta_{0}\right)\right]^{2}} e^{i \nu\left(\theta-\frac{\pi}{2}\right)} .
$$

The last term is the modification of the scattered field introduced by the dielectric. It can also be put into the form,

$$
\psi_{D}^{S C}(\mathbf{r})=\frac{4}{\pi i} \int_{0}^{\infty} \frac{d \nu}{X_{\nu}^{(1)}} \frac{H_{\nu}^{(1)}\left(k_{0} r\right)}{\left[H_{\nu}^{(1)}\left(\beta_{0}\right)\right]^{2}} e^{-i \nu \frac{\pi}{2}} \cos \nu \theta
$$

which converges for all values of $\theta$.

\section{The Surface Distribution}

In this section the distribution of $\psi$ on the dielectric surface will be examined in a number of special cases. This choice of field point does not affect the methods of approximation that will be used, but does simplify the general expression for $\psi$ to a certain extent. For on the circle $r=b$ the first term of (5.7) vanishes, so that the integral to be considered is simply

$$
\psi(\mathbf{b})=\frac{2}{\pi i} \int_{-\infty}^{\infty} \frac{d \nu}{X_{\nu}^{(1)} H_{\nu}^{(1)}\left(\beta_{0}\right)} e^{i \nu\left(\theta-\frac{\pi}{2}\right)} .
$$

In terms of the index of refraction, $n=k / k_{0}$, and the quantity, $\delta=\beta-\alpha$, the conditions under which approximations to (6.1) will be investigated are (a) $\delta / \beta \ll 1$; (b) $|n-1| \ll 1$; and (c) $k_{0} a \gg 1$.

\subsection{Thin Dielectric: $\delta / \beta \ll 1$}

Since all the dielectric effects are contained in the function,

$$
X_{\nu}^{(1)}=\beta \frac{C_{\nu}^{\prime}(\alpha, \beta)}{C_{\nu}(\alpha, \beta)}-\beta_{0} \frac{H_{\nu}^{(1)^{\prime}}\left(\beta_{0}\right)}{H_{\nu}^{(1)}\left(\beta_{0}\right)}
$$

we require the expansion of $1 / X_{\nu}$ when $a \approx b$. It is clearly advantageous to treat $X_{\nu}$ as a function of the variable $\alpha$ (i. e., the inner radius $a$ ) since that parameter appears only in $C_{\nu}$. Moreover, $H_{\nu}^{(1)}\left(\beta_{0}\right)$ has no zeros for real $\nu$, so the first term of $(6.2)$ predominates for small $\delta$. We may 
therefore put $1 / X_{\nu} \approx F_{\nu}$, where

$$
F_{\nu}(\alpha)=\frac{1}{\beta} \frac{C_{\nu}(\alpha, \beta)}{C_{\nu}^{\prime}(\alpha, \beta)}=\frac{1}{\beta} \frac{H_{\nu}^{(1)}(\alpha) H_{\nu}^{(2)}(\beta)-H_{\nu}^{(2)}(\alpha) H_{\nu}^{(1)}(\beta)}{H_{\nu}^{(1)}(\alpha) H_{\nu}^{(2) \prime}(\beta)-H_{\nu}^{(2)}(\alpha) H_{\nu}^{(1) \prime}(\beta)} .
$$

Since $\lim _{\alpha \rightarrow \beta} F_{\nu}(\alpha)=0$, the Taylor expansion of (6.3) has the form

$$
F_{\nu}(\beta-\delta)=-\delta F_{\nu}^{\prime}(\beta)+\frac{1}{2} \delta^{2} F_{\nu}^{\prime \prime}(\beta)-\ldots
$$

With (6.3) and (6.4) it is a straightforward matter to develop the required series explicitly. We need certain relations of the Wronskian type [6], and up to terms of order $\delta^{4}$ the result of the calculation turns out to $\mathrm{be}^{6}$

$$
F_{\nu}(\alpha)=\frac{\delta}{\beta}+\frac{1}{2} \frac{\delta^{2}}{\beta^{2}}+\frac{1}{3} \frac{\delta^{3}}{\beta^{3}}+\frac{1}{4} \frac{\delta^{4}}{\beta^{4}}+\cdots+\frac{1}{3} \frac{\delta^{3}}{\beta^{3}}\left(\beta^{2}-\nu^{2}\right)+\frac{1}{6} \frac{\delta^{4}}{\beta^{4}}\left(2 \beta^{2}-3 \nu^{2}\right)+\ldots
$$

It is clear that the first group of terms represents the function $-\ln [1-(\delta / \beta)]$, so that

$$
\frac{1}{X_{\nu}^{(1)}}=\ln \frac{b}{a}+\frac{1}{3} \frac{\delta^{3}}{\beta^{3}}\left(\beta^{2}-\nu^{2}\right)+\frac{1}{6} \frac{\delta^{4}}{\beta^{4}}\left(2 \beta^{2}-3 \nu^{2}\right)+O\left(\frac{\delta^{5}}{\beta^{5}}\right) .
$$

On substituting (6.6) in (6.1) a series of integrals is obtained, the first of which is

$$
\left(\ln \frac{b}{a}\right) \frac{2}{\pi i} \int_{-\infty}^{\infty} \frac{d \nu}{H_{\nu}^{(1)}\left(\beta_{0}\right)} e^{i \nu\left(\theta-\frac{\pi}{2}\right)}
$$

The calculation of $\partial_{r} \psi_{b}(\mathbf{b})$ from (5.5) shows that except for the constant, $\ln (b / a)$, the last result is just the current distribution on the surface of a perfectly conducting cylinder of radius $b$. Therefore, to a first approximation for thin dielectric sleeves of any refractive index and radius, the electric field distribution on the dielectric surface is proportional to the magnetic field distribution on a conducting cylinder of the same size when illuminated by a plane wave. The first term involving the refractive index is $O(\delta / \beta)^{3}$ and is proportional to $n^{2}$.

\subsection{Low-Refractive Index: $|n-1| \ll 1$}

In order to expand the surface distribution,

$$
\psi(\mathbf{b})=\frac{2}{\pi i} \int_{-\infty}^{\infty} \frac{d \nu}{X_{\nu}^{(1)} H_{\nu}^{(1)}\left(\beta_{0}\right)} e^{i_{\nu}\left(\theta-\frac{\pi}{2}\right)},
$$

in the neighborhood of $k_{0}$, let us consider the function,

$$
G_{\nu}(k)=\left[X_{\nu}^{(1)} H_{\nu}^{(1)}\left(\beta_{0}\right)\right]^{-1} .
$$

Its Taylor expansion may be written in the form

$$
G_{\nu}(k)=G_{\nu}\left(k_{0}\right)+(n-1) k_{0} G_{\nu}^{\prime}\left(k_{0}\right)+O(n-1)^{2},
$$

where $(n-1) k_{0}=k-k_{0}$. Using (6.2) and the Wronskian (2.12), we find at once that

$$
G_{\nu}\left(k_{0}\right)=\frac{\pi i}{4} \frac{C_{\nu}\left(\alpha_{0}, \beta_{0}\right)}{H_{\nu}^{(1)}\left(\alpha_{0}\right)}=\frac{\pi i}{4}\left[H_{\nu}^{(2)}\left(\beta_{0}\right)-\frac{H_{\nu}^{(2)}\left(\alpha_{0}\right)}{H_{\nu}^{(1)}\left(\alpha_{0}\right)} H_{\nu}^{(1)}\left(\beta_{0}\right)\right] .
$$

\footnotetext{
${ }^{6}$ The successive derivatives of $F_{\nu}(\alpha)$, evaluated at $\alpha=\beta$, are

$$
\begin{aligned}
F_{\nu}^{\prime}(\boldsymbol{\beta})=-\frac{1}{\boldsymbol{\beta}} & F_{\nu}^{\prime \prime \prime}(\boldsymbol{\beta})=-\frac{2}{\boldsymbol{\beta}^{3}}\left(1+\boldsymbol{\beta}^{2}-\nu^{2}\right) \\
F_{\nu}^{\prime \prime}(\boldsymbol{\beta})=\frac{1}{\boldsymbol{\beta}^{2}} & F_{\nu}^{\prime \prime \prime \prime}(\boldsymbol{\beta})=\frac{2}{\boldsymbol{\beta}^{4}}\left(3+4 \boldsymbol{\beta}^{2}-6 \nu^{2}\right)
\end{aligned}
$$
}


Thus the first term in the expansion of $(6.1)$ is just $\psi_{a}(\mathbf{b})$, the total field at $r=b$ when the plane wave is scattered by a conducting cylinder of radius $a$ in the medium $k_{0}$.

The second term is found by evaluating

$$
G_{\nu}^{\prime}\left(k_{0}\right)=-H_{\nu}^{(1)}\left(\beta_{0}\right) G_{\nu}^{2}\left(k_{0}\right)\left(\partial_{k} X_{\nu}^{(1)}\right)_{k=k_{0}}
$$

where $\partial_{k}$ signifies the differential operator $\partial / \partial k$. Treating $C_{\nu}$ as a function of two dependent variables $\alpha, \beta$, and replacing $C_{\nu}^{\prime}$ by $\partial_{\beta} C_{\nu}$, we have

$$
\partial_{k} X_{\nu}^{(1)}=\partial_{k}\left[\beta \frac{\partial_{\beta} C_{\nu}(\alpha, \beta)}{C_{\nu}(\alpha, \beta)}\right]
$$

It follows, with the help of (6.9), that

$$
G_{\nu}^{\prime}\left(k_{0}\right)=\frac{\pi^{2}}{16} \frac{H_{\nu}^{(1)}\left(\beta_{0}\right)}{\left[H_{\nu}^{(1)}\left(\alpha_{0}\right)\right]^{2}}\left[b C_{\nu} \partial_{\beta} C_{\nu}+\beta C_{\nu} \partial_{k} \partial_{\beta} C_{\nu}-\beta \partial_{\beta} C_{\nu} \partial_{k} C_{\nu}\right]_{k=k_{0}}
$$

Since $\partial_{k}=a \partial_{\alpha}+b \partial_{\beta}$, the final bracket of (6.11) becomes, after a little rearrangement,

$$
I=k a b\left(C_{\nu} \partial_{\alpha} \partial_{\beta} C_{\nu}-\partial_{\beta} C_{\nu} \partial_{\alpha} C_{\nu}\right)-\beta b\left(\partial_{\beta} C_{\nu}\right)^{2}+b C_{\nu}\left(\partial_{\beta} C_{\nu}+\beta \partial_{\beta}^{2} C_{\nu}\right) .
$$

If we now make use of Bessel's differential equation for $C_{\nu}$, the last parenthesis of (6.12) is seen to be

$$
\partial_{\beta} C_{\nu}+\beta \partial_{\beta}^{2} C_{\nu}=\frac{1}{\beta}\left(\nu^{2}-\beta^{2}\right) C_{\nu}
$$

Moreover, the bracketed expression in the first term of (6.12) can be represented by the Wronskian determinant

$$
W\left(C_{\nu}, \partial_{\beta} C_{\nu}\right)=\left|\begin{array}{ll}
C_{\nu} & \partial_{\beta} C_{\nu} \\
\partial_{\alpha} C_{\nu} & \partial_{\alpha} \partial_{\beta} C_{\nu}
\end{array}\right| .
$$

In the usual way [7] one can show that

$$
\partial_{\beta}(\beta W)=0,
$$

which implies that $\beta W$ is independent of $\beta$. To find the value of the constant we let $\beta=\alpha$. Then $C_{\nu}=0$, and

$$
\lim _{\beta \rightarrow \alpha} \beta W=-\alpha\left(\partial_{\alpha} C_{\nu} \partial_{\beta} C_{\nu}\right)_{\beta=\alpha}
$$

In the limit the derivatives are ordinary Wronskians like (2.12), and it follows that

$$
W=-\frac{16}{\pi^{2} \alpha \beta} .
$$

The result of substituting (6.13) and (6.15) into (6.12) is

$$
I=-\frac{16}{\pi^{2} k}-\frac{1}{k}\left(\beta \partial_{\beta} C_{v}\right)^{2}-\frac{1}{k}\left(\beta^{2}-\nu^{2}\right) C_{\nu}^{2}
$$

With this result, eq (6.8), (6.9), and (6.11) can be combined into an explicit expression for the first term of the expansion of $G_{\nu}(k)$ near $k=k_{0}$. Substitution in (6.1) yields the following perturbed distribution at $r=b$ when $n \approx 1$ :

$$
\begin{gathered}
\psi(\mathbf{b}) \approx \psi_{\alpha}(\mathbf{b})-(n-1)\left\{\frac{2}{\pi i} \int_{-\infty}^{\infty} \frac{H_{\nu}^{(1)}\left(\beta_{0}\right)}{\left[H_{\nu}^{(1)}\left(\alpha_{0}\right)\right]^{2}} e^{i \nu\left(\theta-\frac{\pi}{2}\right)} d \nu+\frac{\pi}{8 i} \int_{-\infty}^{\infty}\left[\frac{\beta_{0} C_{\nu}^{\prime}\left(\alpha_{0}, \beta_{0}\right)}{H_{\nu}^{(1)}\left(\alpha_{0}\right)}\right]^{2} H_{\nu}^{(1)}\left(\beta_{0}\right) e^{i \nu\left(\theta-\frac{\pi}{2}\right)} d \nu\right. \\
\left.+\frac{\pi}{8 i} \int_{-\infty}^{\infty}\left(\beta_{0}^{2}-\nu^{2}\right)\left[\frac{C_{\nu}\left(\alpha_{0}, \beta_{0}\right)}{H_{\nu}^{(1)}\left(\alpha_{0}\right)}\right]^{2} H_{\nu}^{(1)}\left(\beta_{0}\right) e^{i \nu\left(\theta-\frac{\pi}{2}\right)} d \nu\right\} .
\end{gathered}
$$


If one is interested in the perturbation introduced by the dielectric at points outside the sleeve, it is only necessary to put $H_{\nu}^{(1)}\left(k_{0} r\right)$ for $H_{\nu}^{(1)}\left(\beta_{0}\right)$ wherever that function appears explicitly in (6.16). Whether the field point is on the surface or outside the sleeve, however, it is readily verifiable that all the integrals converge and that the bracket vanishes when $b=a$, leaving only the field of the conducting cylinder. In general, it seems that none of the correction terms in (6.16) can be neglected.

\subsection{Very-High Frequencies: $k_{0} a \gg 1$}

The last method of approximation to be dealt with is useful when both inner and outer cylinders are large compared to the wavelength in either medium. By combining the longer wavelength with the shorter radius these conditions are satisfied by the single relation, $\alpha_{0} \gg 1$. When this requirement is met, no further restrictions need be imposed, a priori, on either the thickness of the dielectric sleeve or on its refractive index. Since we shall consider only the contribution to (6.1) from that part of the integral for which $|\nu|+\left.3{ }_{\mid \nu}\right|^{1 / 3}<\alpha_{0}$, it is possible to use the Debye approximation for all Hankel functions. To this order of accuracy we may expect that the integration will yield the optical rays, but no diffraction effects.

For a typical Hankel function the appropriate asymptotic form is

$$
H_{\nu}^{(1)}\left(\beta_{0}\right) \sim 2^{1 / 2} e^{-i \frac{\pi}{4}}\left(\pi \beta_{0} \sin \eta_{0}\right)^{-1 / 2} \exp i \beta_{0} f\left(\eta_{0}\right),
$$

where $\eta_{0}$ is the real angle

and

$$
\eta_{0}=\cos ^{-1}\left(\nu / \beta_{0}\right)
$$

$$
f\left(\eta_{0}\right)=\sin \eta_{0}-\eta_{0} \cos \eta_{0} .
$$

The corresponding approximation for the derivative with respect to the argument, $\beta_{0}$, is

$$
H_{\nu}^{(1) \prime}\left(\beta_{0}\right) \sim i \sin \eta_{0} H_{\nu}^{(1)}\left(\beta_{0}\right) .
$$

With (6.17) and (6.20) it follows that

$$
\beta_{0} \frac{H_{\nu}^{(1) \prime}\left(\beta_{0}\right)}{H_{\nu}^{(1)}\left(\beta_{0}\right)} \sim i \beta_{0} \sin \eta_{0}
$$

In a similar way, by defining the angles

and the function

$$
\eta=\cos ^{-1}(\nu / \beta) ; \quad \zeta=\cos ^{-1}(\nu / \alpha),
$$

$$
F(\nu)=\beta f(\eta)-\alpha f(\zeta)
$$

one can show that

$$
\beta \frac{C_{\nu}^{\prime}(\beta)}{C_{\nu}(\beta)} \sim i \beta \sin \eta \frac{e^{i F(\nu)}+e^{-i F(\nu)}}{e^{i F(\nu)}-e^{-i F(\nu)}} .
$$

If (6.21) and (6.24) are now combined according to (6.2), we find that

$$
\frac{1}{X_{\nu}} \sim \frac{1}{\beta_{0} \sin \eta_{0}} \frac{i \rho}{1+\rho} \frac{1-e^{2 i F^{(\nu)}}}{1+\tau e^{2 i F(\nu)}}
$$

where $\rho$ and $\tau$ signify the ratios,

$$
\rho=\frac{\beta_{0} \sin \eta_{0}}{\beta \sin \eta} ; \quad \tau=\frac{1-\rho}{1+\rho} .
$$

Now for all $\nu^{2}<\beta_{0}^{2}, \beta \sin \eta>\beta_{0} \sin \eta_{0}$, so that $0>\rho>1$. Consequently, $0<\tau<1$ and $|\tau \exp 2 i F(\nu)|<1$. The denominator of (6.25) can therefore be represented by its Taylor expansion, and it follows that

$$
\frac{1}{X_{\nu}^{(1)}} \sim \frac{1}{\beta_{0} \sin \eta_{0}} \frac{i \rho}{1+\rho}\left[1-(1+\tau) e^{2 i F^{(\nu)}}+(1+\tau) \tau e^{4 i F(\nu)}-(1+\tau) \tau^{2} e^{6 i F^{(\nu)}}+\ldots\right]
$$


This result already makes evident the ray nature of the approximation. Successive terms of the series correspond to the rays which are, (1) reflected by the sleeve, (2) reflected once at the conducting cylinder, (3) reflected once at the conducting cylinder and once internally at the dielectric surface, etc. The phase function of each term corresponds to the number of path segments a ray traverses in the dielectric (with a minus sign added for each reflection at the conducting cylinder), and its amplitude function includes the appropriate transmission and reflection coefficients for each contact with the dielectric surface. Since these quantities are all functions of the integration variable, successive terms can be evaluated in detail only by more and more intricate stationary phase approximations. There is, however, one limiting case for which it is relatively easy to establish the correspondence between the terms of (6.27) and optical rays. When the radii $a$ and $b$ become indefinitely large, while their difference, $d=b-a$, remains constant, the solution for the cylindrical problem at each point $\mathbf{b}=(b, \theta)$ of the dielectric surface approaches that for a plane wave scattered by a conducting plane sheet with a dielectric coating of thickness $d$, the dielectric surface being tangent to the cylinder at the point in question.

To see how this comes about let us substitute (6.27) and (6.17) in (6.1). The first integral of the resulting asymptotic series becomes

$$
\psi^{(1)}(\mathbf{b})=\left(\frac{2}{\pi}\right)^{1 / 2} e^{i \frac{\pi}{4}} \int_{-A}^{A} d \nu\left(\beta_{0} \sin \eta_{0}\right)^{-1 / 2} \frac{\rho}{1+\rho} \exp i g^{(1)}(\nu)
$$

where the limits on the integral define the interval within which the Debye approximations are valid and where

$$
g^{(1)}(\nu)=-\beta_{0} f\left(\eta_{0}\right)+\nu \theta-\frac{1}{2} \nu \pi, \quad 0<\theta
$$

For large $\beta_{0}$ the principal contributions to this integral come from the neighborhood of the stationary points of $g^{(1)}(\nu)$ in $(-A, A)$. Since the asymptotic integrand decays exponentially outside this interval, one may extend its limits to $(-\infty, \infty)$ without appreciable error. Then if a stationary point of $g^{(1)}(\nu)$ is located at $\nu=\nu_{1}$, the Taylor expansion of (6.28) in the vicinity of this point gives as its first approximation,

$$
\psi^{(1)}(\mathbf{b}) \sim\left(\frac{2}{\pi \beta_{0}}\right)^{1 / 2}\left(\sin \eta_{0}\right)_{1}^{-1 / 2} \frac{\rho_{1}}{1+\rho_{1}} e^{i \frac{\pi}{4}} \int_{-\infty}^{\infty} d \nu \exp i\left[g^{(1)}\left(\nu_{1}\right)+\frac{\lambda^{2}}{2} \partial_{\nu}^{2} g^{(1)}\left(\nu_{1}\right)\right]
$$

where $\lambda=\nu-\nu_{1}$ and the subscripts indicate functions that are to be evaluated at $\nu=\nu_{1}$.

The value of $\nu$ required in (6.30) is that which reduces $\partial_{\nu} g^{(1)}\left(\nu_{1}\right)=0$ to an identity. It can be shown that this occurs for the angle $\left(\eta_{0}\right)_{1}=\frac{1}{2} \pi-\theta$, corresponding to

$$
\nu_{1}=\beta_{0} \sin \theta
$$

According to the condition specified prior to (6.17), the angle $\theta$ must therefore be such that

$$
\sin \theta+3 \beta^{-2 / 3}(\sin \theta)^{1 / 3}<\frac{\alpha_{0}}{\beta_{0}}
$$

It is clear that this inequality cannot under any circumstances be satisfied at the shadow boundary where $\theta=\pi / 2$. With (6.31) and (6.26) we determine that

and

$$
\partial_{\nu}^{2} g^{(1)}\left(\nu_{1}\right)=-\frac{1}{\beta_{0} \cos \theta}
$$

$$
\frac{2 \rho_{1}}{1+\rho_{1}}=\frac{2 \beta_{0} \cos \theta}{\beta_{0} \cos \theta+\beta \cos \theta_{n}}=1+R,
$$

where $R$ is the reflection coefficient for a plane dielectric surface [8] and the angles of incidence 
and refraction are related by

With these results, we have

$$
\frac{\sin \theta}{\sin \theta_{n}}=n
$$

$$
\psi^{(1)}(\mathbf{b}) \sim \frac{1}{2}(1+R) e^{-i \beta_{0} \cos \theta}\left[\frac{2}{\pi \beta_{0} \cos \theta}\right]^{1 / 2} e^{i \frac{\pi}{4}} \int_{-\infty}^{\infty} d \lambda \exp \left[-i \frac{\lambda^{2}}{2 \beta_{0} \cos \theta}\right] .
$$

The value of the infinite integral is given in standard tables, and (6.34) can be reduced to

$$
\psi^{(1)}(\mathbf{b}) \sim(1+R) e^{-i \beta_{0} \cos \theta},
$$

which corresponds in amplitude and phase to the sum of the incident and reflected rays at the dielectric interface for angles $\theta<\frac{\pi}{2}$ (fig. 3). Note that further restrictions on $\theta$, such as (6.32), are no longer required.

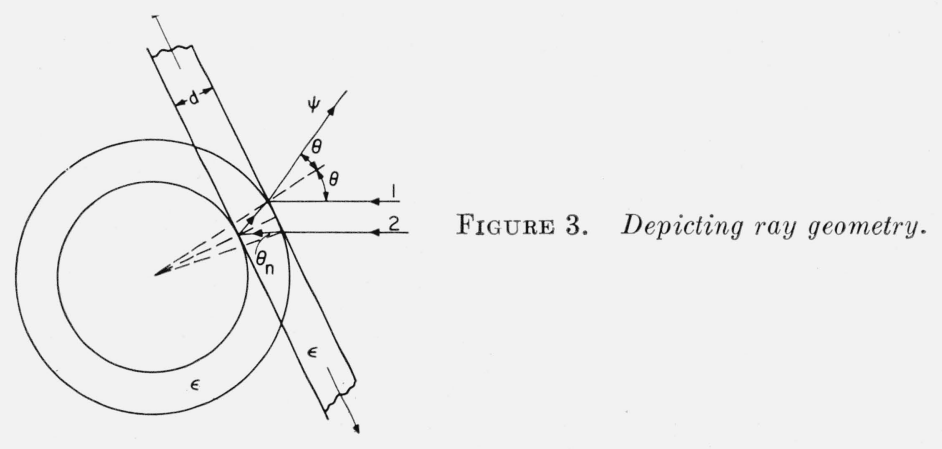

The second term in the expansion of (6.1) is

where

$$
\psi^{(2)}(\mathbf{b})=-\left(\frac{2}{\pi}\right)^{1 / 2} e^{i \frac{\pi}{4}} \int_{-A}^{A} d \nu\left(\beta_{0} \sin \eta_{0}\right)^{-1 / 2} \frac{2 \rho}{(1+\rho)^{2}} \exp i g^{(2)}(\nu),
$$

$$
g^{(2)}(\nu)=2 F(\nu)+g^{(1)}(\nu) .
$$

Again, after finding the point $\nu_{2}$ at which $\partial_{\nu} g^{(2)}\left(\nu_{2}\right)$ vanishes, we can approximate (6.36) with

$$
\psi^{(2)}(\mathbf{b}) \sim-\left(\frac{2}{\pi \beta_{0}}\right)^{1 / 2}\left(\sin \eta_{0}\right)_{2} \frac{2 \rho_{2}}{\left(1+\rho_{2}\right)^{2}} e^{i \frac{\pi}{4}} \int_{-\infty}^{\infty} d \lambda \exp i\left[g^{(2)}\left(\nu_{2}\right)+\frac{\lambda^{2}}{2} \partial_{\nu}^{2} g^{(2)}\left(\nu_{2}\right)\right]
$$

where $\lambda=\nu-\nu_{2}$ and the subscripts indicate functions that are to be evaluated at $\nu=\nu_{2}$.

According to (6.37) the required stationary point is the solution of

$$
\partial_{\nu}\left[2 F(\nu)+g^{(1)}(\nu)\right]=0 .
$$

With the help of (6.23) and (6.29) it may be shown that this equation is equivalent to

$$
-2(\eta-\zeta)+\eta_{0}=\frac{1}{2} \pi-\theta
$$

In order to discuss the solution of (6.40) in the plane limit we shall assume that $(\eta-\zeta)$ is small so that $\sin (\eta-\zeta)$ is approximately equal to its argument. Then

$$
\eta-\zeta \approx \frac{\cos \eta}{\sin \eta} \frac{\delta}{\beta}
$$


where $\delta=k d$ and terms of order $(\delta / \beta)^{2}$ have been neglected. Accordingly, if $(\eta-\zeta)$ is to be a small quantity it is sufficient that $\delta / \beta=d / b<<1$, provided $\eta \neq 0$ or what amounts to the same thing, $\nu^{2} \neq \beta^{2}$. This requirement is, of course, the same as that already imposed by the original Debye approximations. With this understanding, (6.40) can be put into the more convenient form

$$
\cos \left[\eta_{0}-2 \frac{\cos \eta}{\sin \eta} \frac{\delta}{\beta}\right]=\sin \theta
$$

Expanding the left-hand side of this relation up to terms of order $\delta / \beta$, we obtain

$$
\nu_{2}-\nu_{1}=-2 \rho_{1} \nu_{1} \frac{\delta}{\beta},
$$

where as before

$$
\nu_{1}=\beta_{0} \sin \theta ; \quad \rho_{1}=\frac{\beta_{0} \cos \theta}{\beta \cos \theta_{n}} .
$$

Since in this limit $\nu_{2}$ is a perturbation on $\nu_{1}$, all functions of $\nu_{2}$ in (6.38) have Taylor expansions in the neighborhood of $\nu_{1}$. Retaining terms in $\delta / \beta$ if they affect the phase of $\psi^{(2)}$ but not otherwise, we find, for example, that

$$
\begin{aligned}
g^{(2)}\left(\nu_{2}\right) & =g^{(2)}\left(\nu_{1}\right)+\left(\nu_{2}-\nu_{1}\right) g^{(2) \prime}\left(\nu_{1}\right)+\ldots \\
& =-\beta_{0} \cos \theta+2\left(\beta \cos \theta_{n}\right) \frac{\delta}{\beta}+O\left(\frac{\delta}{\beta}\right)^{2} .
\end{aligned}
$$

To this degree of approximation (6.38) becomes

$\psi^{(2)}(\mathbf{b}) \sim-\frac{1}{2}(1+R)(1-R)\left[\frac{2}{\pi \beta_{0} \cos \theta}\right]^{\frac{1}{2}} \exp \left[-i \beta_{0} \cos \theta+2 i \delta \cos \theta_{n}\right] e^{i \frac{4}{\pi}} \int_{-\infty}^{\infty} d \lambda \exp \left[-i \frac{\lambda^{2}}{2 \beta_{0} \cos \theta}\right)$,

and we finally obtain

where

$$
\psi^{(2)}(\mathbf{b}) \sim(1+R)(1-R) \exp \left[-i \beta_{0} \cos \theta+2 i \delta \cos \theta_{n}\right],
$$

$$
1-R=\frac{2 \beta \cos \theta_{n}}{\beta_{0} \cos \theta+\beta \cos \theta_{n}}=T_{n}
$$

The quantity, $T_{n}$, is the transmission coefficient of a ray incident on the dielectric surface from the interior at an angle $\theta_{n}$ with the normal. If the transmission coefficient from the external region is designated by $T=1+R$, then the sum of (6.35) and (6.46) has the form

$$
\psi(\mathbf{b}) \sim e^{-i \beta_{0} \cos \theta}\left[T-T T_{n} e^{2 i \delta \cos \theta_{n}}+\ldots\right] .
$$

These two rays are depicted in figure 3. It is an elementary exercise in geometry to show that their amplitudes and phases are exactly those predicted by ray optics, provided the dielectric surface is nearly plane over the arc segment illuminated by the bundle included between rays 1 and 2. Additional rays for this quasi-plane example may be written down by inspection from the further terms of $(6.27)$.

\section{References}

[1] C. C. Tang, J. Appl. Phys. 28, 628 (1957).

[2] E. C. Titchmarsh, Eigenfunction expansions, p. 113-16 (Oxford University Press, Oxford, England, 1958).

[3] N. Marcuvitz, Commun. Pure Appl. Math. 4, 284 (1951).

[4] T. T. Wu, Phys. Rev. 104, 1201 (1956).

[5] R. D. Kodis, Brown Univ. Sci. Rpt. 1391/8, AFCRC-TN-57-957 (October 1957).

[6] G. N. Watson, Bessel functions, p. 76, 2d ed. (Cambridge University Press, New York 22, N.Y., 1945).

[7] G. N. Watson, Bessel functions, p. 42, 2d ed. (Cambridge University Press, New York 22, N.Y., 1945).

[8] J. A. Stratton, Electromagnetic theory, p. 492-4, 1st ed. (McGraw-Hill Book Co., Inc., New York, N.Y., 1941).

(Paper 65D1-99) 